\title{
OBSTÁCULOS AL DESARROLLO \\ DE LAS PEQUEÑAS Y MEDIANAS EMPRESAS EN AMÉRICA LATINA*
}

\author{
Emilio Zevallos V. \\ FUNDACIÓN PARA EL DESARROLLO SOSTENIBLE \\ EN AMÉRICA LATINA \\ ezevallos@fundes.org
}

\section{Resumen}

Este documento es un avance de investigación comparada sobre el entorno, los obstáculos y las perspectivas de las pequeñas y medianas empresas en ocho países de América Latina, aunque en este artículo sólo se trabaja con seis de ellos: Argentina, Colombia, Costa Rica, Chile, El Salvador y Panamá. En la primera parte se presenta una breve contextualización de cada país en términos de conceptos, universo empresarial y áreas consideradas problemáticas. También se intenta establecer una tipología general de la empresa. En la segunda parte se reseñan los aspectos metodológicos del estudio, mientras que en la tercera se muestran las condiciones del entorno de los negocios en cada país, segmentándolo por áreas (para este artículo sólo financiamiento, recursos humanos y tecnología) y por actores (sólo empresarios pyme). Finalmente, se ofrecen algunas reflexiones respecto a la situación de la pequeña y mediana empresa y se esbozan líneas de acción en términos de políticas públicas.

Palabras clave: pymes, América Latina, entorno, empresas.

\begin{abstract}
This document reports progress made in compared research about the environment, barriers and prospects facing small and intermediate size companies in eight Latin American countries, although only six are presented in this paper, namely Argentina, Colombia, Costa Rica, Chile, El Salvador and Panama. The first section briefly presents each country's business environment in terms of prevailing concepts, the overall business universe and its trouble spots. It also aims at establishing a general typology of companies. The second section reviews the study's methodology while the third chapter deals with the elements for doing business in each country (this paper deals only with the financing, human resources and technology components). Finally, it presents some reflections about the condition of small and intermediate companies and some guidelines for public policy actions.
\end{abstract}

Key words: small and intermediate companies (SMEs), Latin America, environment, companies.

* Este documento es parte de una investigación más amplia del autor sobre las condiciones del entorno de los negocios en ocho países de América Latina. 


\section{Introducción}

Las pequeñas y medianas empresas (pymes) son objeto, de manera creciente, de numerosas investigaciones y análisis tanto en países industrializados como en desarrollo. La progresiva importancia de las pymes en el proceso de crecimiento económico de las naciones se debe, fundamentalmente, al empleo que generan en un contexto donde conviven dos situaciones: por un lado, una creciente demanda de empleo por parte de los jóvenes (de baja calificación) que cada año se integran a la población económicamente activa (PEA) y, por el otro, la imposibilidad del sector moderno de la economía de absorber esa creciente demanda. Todo ello en un escenario donde la innovación y la mayor calificación laboral son variables clave para mantener la competitividad de las empresas.

Frente a este panorama, la pyme aparece como un potencial elemento dinamizador de la economía en la medida en que, en comparación con la microempresa, genera empleo de mayor calidad, en cuanto a estabilidad e ingresos; utiliza mayor capital; cuenta con más infraestructura; tiene mayor potencial de integrarse en cadenas productivas, debido a su mayor stock de capital y uso de tecnología; y por lo tanto, su productividad relativa es más alta.

Dados estos antecedentes, el objetivo del presente avance de investigación es presentar algunos resultados de un diagnóstico comparado de la situación de las pymes en ocho países de América Latina. En él se identifican -en una perspectiva regional- los obstáculos más importantes al desarrollo competitivo de las pymes, se analizan las razones que se encuentran detrás de ellos y se establece una caracte- rización tanto de las pequeñas y medianas empresas de la región como de la forma de encarar tales barreras a favor de su competitividad.

El diagnóstico se basa en estudios y bases de datos previamente desarrollados por Fundes ${ }^{1}$ en ocho países: Argentina, Bolivia, Colombia, Costa Rica, Chile, El Salvador, Panamá y Venezuela, aunque los considerados para el presente artículo son únicamente seis: Argentina, Colombia, Costa Rica, Chile, El Salvador y Panamá. En este punto, vale la pena señalar que en esta parte de la investigación se entrevistaron a más de 2700 empresarios en los seis países (un promedio superior a 450 empresarios por país). El estudio abarca siete áreas problemáticas para las pymes: financiamiento, recursos humanos, tecnología e información, comercio exterior, estructura regulatoria y competencia, organización y cooperación empresarial, y temas particulares. En este avance sólo analizaremos tres de ellos: financiamiento, recursos humanos, y tecnología e información. Esto debido a la relevancia de estos temas, así como a su representatividad regional.

Cabe señalar que la investigación final abarca tres perspectivas: la de los propios empresarios propietarios de pequeñas y medianas empresas, la de los gobiernos y la de las organizaciones intermedias (instituciones académicas, gremios empresariales, entre otras). Sin embargo, por razones de espacio, en este avance sólo se considera la opinión de los empresarios, que es el eje de la investigación.

1. Fundes, la Fundación para el Desarrollo Sostenible en América Latina, es una organización del sector privado dedicada a la promoción del desarrollo de la pequeña y mediana empresa. 
En la primera parte se presenta información general sobre las pequeñas y medianas empresas en los países analizados, así como el contexto en el que desenvuelven su actividad. La segunda parte señala brevemente algunos aspectos metodológicos del estudio. Más adelante se analiza en detalle la problemática general y la visión empresarial de las tres áreas consideradas. Finalmente, cierran el artículo las conclusiones preliminares del estudio y algunas propuestas de líneas de trabajo.

\section{Información general y tipología}

Como una primera aproximación al mundo de la pequeña y mediana empresa, es importante señalar alguna información básica de los países de la región involucrados en el estudio. En ellos se emplean diferentes criterios para definir qué es una pyme: número de trabajadores, ventas, activos, así como combinaciones de varios de estos. En algunos países se usa más de un criterio, mientras en otros se encuentran varias definiciones, dependiendo de

\section{Cuadro 1. Definiciones}

\begin{tabular}{|c|c|c|c|c|c|}
\hline Países & Criterios & Microempresa & $\begin{array}{l}\text { Pequeña } \\
\text { empresa }\end{array}$ & $\begin{array}{l}\text { Mediana } \\
\text { empresa }\end{array}$ & $\begin{array}{c}\text { Gran } \\
\text { empresa }\end{array}$ \\
\hline Argentina $^{1}$ & Ventas & $\begin{array}{l}\text { Hasta medio } \\
\text { millón de } \\
\text { pesos }\end{array}$ & $\begin{array}{l}\text { Hasta } 3 \\
\text { millones } \\
\text { de pesos }\end{array}$ & $\begin{array}{l}\text { Hasta } 24 \\
\text { millones } \\
\text { de pesos }\end{array}$ & $\begin{array}{l}\text { Más de } 24 \\
\text { millones } \\
\text { de pesos }\end{array}$ \\
\hline Chile $^{2}$ & Ventas & Hasta 2400 UF & $\begin{array}{l}\text { Hasta } 25 \\
\text { mil UF }\end{array}$ & $\begin{array}{l}\text { Hasta } 100 \\
\text { mil UF }\end{array}$ & $\begin{array}{l}\text { Más de } 100 \\
\text { mil UF }\end{array}$ \\
\hline Colombia $^{3}$ & Empleo & $\begin{array}{l}\text { Hasta } 10 \\
\text { puntos }\end{array}$ & $\begin{array}{l}\text { Hasta } 50 \\
\text { puntos }\end{array}$ & $\begin{array}{l}\text { Hasta } 200 \\
\text { puntos }\end{array}$ & $\begin{array}{l}\text { Más de } 200 \\
\text { puntos }\end{array}$ \\
\hline Costa Rica ${ }^{4}$ & $\begin{array}{l}\text { Empleo, ventas } \\
\text { y activos }\end{array}$ & $P \leq 10$ & $10<P \leq 35$ & $35<P \leq 100$ & $P>100$ \\
\hline El Salvador ${ }^{5}$ & Empleo & $\begin{array}{l}\text { Hasta } 4 \\
\text { trabajadores }\end{array}$ & $\begin{array}{l}\text { Hasta } 49 \\
\text { trabajadores }\end{array}$ & $\begin{array}{l}\text { Hasta } 99 \\
\text { trabajadores }\end{array}$ & $\begin{array}{l}\text { Más de } 99 \\
\text { trabajadores }\end{array}$ \\
\hline Panamá 6 & Ingresos & $\begin{array}{l}\text { Hasta } 150 \\
\text { mil dólares }\end{array}$ & $\begin{array}{l}\text { Hasta un } \\
\text { millón de } \\
\text { dólares }\end{array}$ & $\begin{array}{l}\text { Hasta } 2,5 \\
\text { millones de } \\
\text { dólares }\end{array}$ & $\begin{array}{l}\text { Más de } 2,5 \\
\text { millones de } \\
\text { dólares }\end{array}$ \\
\hline
\end{tabular}

1. Definición para manufactura, además hay otras para comercio y servicios, también en ventas anuales.

2. En unidades de fomento (UF). Una UF es aproximadamente US\$ 33 (agosto 2006). También hay otra definición por empleo.

3. Recientemente, se reglamentó la ley sobre pequeña y mediana empresa que establece un puntaje para la definición del tamaño de las empresas. El puntaje se obtiene de una ponderación de tres variables: el valor de las ventas anuales, el valor de los activos fijos y el personal promedio empleado. Este puntaje se aplica de manera diferente para la manufactura, el comercio y los servicios.

4. Donde $\mathrm{P}$ es una ponderación de empleo, activos y ventas anuales.

5. Las definiciones oficiales son por empleos y activos (Conamype).

6. En balboas o dólares (paridad uno a uno).

Elaboración: Equipos Entorno Países de Fundes, con información de la Sepyme, de Argentina; Casen y Corfo, de Chile; Ley MiPyme, julio 2000, de Colombia; Ministerio de Economía de Costa Rica; Dirección General de Estadística y Censos de El Salvador; Ley 33 y Ley 8 del 2000, de Panamá. 
si la unidad económica es manufacturera, comercial o de servicios. Si bien del grupo de países analizados no puede establecerse un criterio general, estudios más amplios en el ámbito latinoamericano nos permiten señalar que la variable más recurrente para definir los estratos empresariales es el empleo, o número de trabajadores.

Sobre la base de tales definiciones se llevó a cabo la cuantificación. Debe resaltarse que la mayor parte de esta información data de la segunda mitad de los años noventa (salvo para Colombia y Argentina). Asimismo, en algunos casos la información corresponde sólo a la manufactura, mientras que en otros se consideran muestras, de cierta forma, representativas; incluso algunos países únicamente señalan información de empresas con cierto tipo de registro. Todo ello lleva a reconocer la presencia de cierta subestimación en el número de unidades económicas. No obstante, este es un primer punto de referencia de la realidad de las empresas en la región.

En sentido estricto, y utilizando las definiciones oficiales, las pequeñas y medianas empresas en estos seis países representan, en promedio, el $11 \%$ de todas las unidades económicas. En el ámbito de la región, sin embargo, este estrato empresarial varía en el rango comprendido entre el $5 \%$ y el $17 \%$.

De otro lado, la distribución geográfica y sectorial de las empresas ayuda a identificar las concentraciones empresariales y las actividades económicas preponderantes. Tanto esta como otras investigaciones revelan una alta concentración empresarial en las capitales de los países, así como en las ciudades más importantes. Este hecho se asociaría a las economías de aglomeración que se generan en las grandes ciudades, con impacto en los

Cuadro 2. Número de empresas

\begin{tabular}{lcccccr}
\hline \multicolumn{1}{c}{ País } & $\begin{array}{c}\text { Año de la } \\
\text { información }\end{array}$ & Microempresa & $\begin{array}{c}\text { Pequeña } \\
\text { empresa }\end{array}$ & $\begin{array}{c}\text { Mediana } \\
\text { empresa }\end{array}$ & $\begin{array}{c}\text { Gran } \\
\text { empresa }\end{array}$ & Total \\
\hline Argentina $^{1}$ & 1994 & 814400 & 69500 & 7400 & 5200 & 896500 \\
Chile $^{2}$ & 1997 & 432431 & 78805 & 10870 & 4814 & 526920 \\
Colombia $^{3}$ & 1990 & 657952 & 26694 & & 821 & 685467 \\
Costa Rica $^{4}$ & 2000 & 58620 & 14898 & & 1348 & 74866 \\
El Salvador & 1998 & 464000 & 12398 & 502 & 316 & 477216 \\
Panamá6 & 1998 & 34235 & 5601 & 1149 & 1239 & 42224 \\
\hline
\end{tabular}

1. Son 903995 incluyendo las denominadas empresas auxiliares. Datos del Indec, Censo Nacional Económico.

2. Datos de la Corporación de Fomento de la Producción (Corfo).

3. Datos del Dane, Censo Económico. Sin embargo, otras fuentes señalan que para 1991 había casi un millón de empresas (Econometría Ltda.).

4. No hay desglose entre pequeña y mediana empresa. Datos de Castillo y Chaves (2001).

5. Sólo industria, comercio y servicios. Datos del Ministerio de Economía y Conamype, Encuesta Económica Anual y Encuesta Microempresarial.

6. Datos del Directorio de Establecimientos, Contraloría de la República.

Elaboración: Equipos Entorno Países de la red Fundes, con base en las fuentes citadas. 
Cuadro 3. Regiones (estados o departamentos) que concentran el $50 \%$ de las empresas (o más)

\begin{tabular}{lcc}
\hline \multicolumn{1}{c}{ Países } & Porcentaje & $\begin{array}{c}\text { Número de regiones } \\
\text { respecto al total }\end{array}$ \\
\hline Argentina $^{1}$ & 55,2 & 3 de 20 \\
Chile $^{2}$ & 48,5 & 2 de 13 \\
Colombia $^{3}$ & 49,0 & 1 de 7 \\
Costa Rica $^{4}$ & 74,8 & 1 de 6 \\
El Salvador & 55,3 & 2 de 14 \\
Panamá6 & 52,4 & 1 de 9 \\
\hline
\end{tabular}

1. Capital Federal + Gran Buenos Aires + Resto Buenos Aires.

2. Metropolitana y Biobío.

3. Sólo Bogotá (le siguen Valle con $15 \%$ y Antioquia con $14 \%$ ).

4. Región central.

5. San Salvador y Santa Ana (luego La Libertad con $11,4 \%$ y San Miguel con 10,6\%)

6. Sólo Panamá (le sigue Chiriquí con $14,8 \%$ )

Elaboración: Fundes, con base en la información recopilada por los equipos Entorno Países de la red Fundes.

Cuadro 4. Participación sectorial de las empresas (en \%)

\begin{tabular}{lccc} 
& Manufactura & Comercio & Servicios \\
\hline Argentina & 13 & 52 & 36 \\
Colombia $^{1}$ & 26 & 31 & 43 \\
Chile $^{2}$ & 11 & 41 & 16 \\
Costa Rica $^{3}$ & 19 & 33 & 48 \\
El Salvador & 11 & 62 & 27 \\
Panamá & 8 & 55 & 36 \\
\hline
\end{tabular}

Nota: En algunos casos las columnas pueden no sumar 100 porque los países tal vez consideren otros sectores no incluidos aquí.

1. Sólo para pymes.

2. En el rubro servicios sólo se consideran servicios personales y restaurantes. No se toman en cuenta otros sectores.

3. El rubro servicios incluye transportes y comunicaciones y es sólo para pymes.

Elaboración: Fundes, con base en la información recopilada por los equipos Entorno Países a partir de información oficial o estudios del sector.

costos de producción de muchas actividades económicas. La evidencia presentada en el cuadro 3 muestra más un fenómeno generalizado que casos particulares. Este patrón se repite tanto en los países de América Latina como en las economías industrializadas.
Nuestra propia evidencia señala que las pymes en la región latinoamericana se encuentran menos concentradas que las grandes empresas, de ahí su importancia como factor de descentralización de oportunidades y como promotor del desarrollo regional. 
Por sectores, se observa una alta concentración en el ramo comercial y de servicios para la mayor parte de los países con información disponible.

En promedio, podemos decir que en estos países la composición sectorial le otorga a la manufactura el $15 \%$; al comercio, el $46 \%$; y a los servicios, el $34 \%$. Algunas de las razones de tal composición son las condiciones del entorno, la normativa, y los propios requerimientos de habilidades y calificación en la rama manufacturera respecto de actividades como el comercio o los servicios.

Este set de información determina el contexto del análisis. A ello se suma la problemática de las empresas, que se revela como otro complejo e interrelacionado conjunto de ámbitos o áreas, donde tanto los empresarios como otros actores vinculados al campo empresarial han señalado conflictos. Con fines de simplificación, las siguientes áreas se han considerado como «relevantes» o «problemáticas»:

a) El área financiera.

b) La infraestructura (y los servicios públicos).

c) La política, que incluye tanto la gestión macroeconómica, el manejo de los temas tributario y laboral, el análisis de las políticas y acciones de fomento, así como el funcionamiento del Estado y las instituciones.

d) Los problemas sociales (y culturales).

e) El mercado interno.

f) El comercio exterior.

g) La tecnología y la información.

h) El medio ambiente.

Cada área presenta un cúmulo de especificidades asociadas a sus contextos nacionales, pero también elementos comunes que pueden ser generalizados. Los temas de financiamiento, recursos humanos, y tecnología e información son una buena prueba de ello; de manera que este avance de investigación se concentra en estas áreas.

\section{Aspectos metodológicos}

Como referencia general, los países donde se desarrollaron los estudios comprenden una superficie de más de 6,8 millones de $\mathrm{km}^{2}$, el $33 \%$ de la superficie de América Latina y el Caribe, y tienen en conjunto más de 139 millones de habitantes, el $27 \%$ de la población de nuestra región. Desde la perspectiva del Índice de Desarrollo Humano del Programa de las Naciones Unidas para el Desarrollo (UNDP, 2004: 139-141), en este grupo existen países con un alto desarrollo humano, como Argentina, Costa Rica y Chile (puestos 34, 45 y 43, respectivamente), junto a países de desarrollo humano medio como Panamá (61), Colombia (73), Venezuela (68), El Salvador (103) y Bolivia (114), lo que señala cierta representatividad respecto de la realidad regional.

Según la Cepal (2003), la región contaba con un PBI per cápita promedio de 3280 dólares al año 2002, aunque en el caso de los países analizados, este indicador varía desde los 900 dólares de Bolivia hasta los 4260 dólares de Chile.

El estudio se desarrolla tanto en las capitales como en otras grandes ciudades de los países mencionados, conforme se encuentra distribuida la población empresarial en ellos. En términos generales se consideran unidades económicas comerciales, de servicios y de manufactura, pero 
se incorporan otros sectores en función de su importancia relativa en los países.

Cálculos someros sobre la información disponible señalan que en los países analizados probablemente existan entre 3,5 y 4 millones de unidades económicas, de las cuales por lo menos 250 mil serían pymes (según las definiciones oficiales y/ o más frecuentes de cada país). A partir de la información disponible para América Latina, podemos inferir que aproximadamente hay 15 millones de unidades económicas, de las cuales un millón serían pymes.

\subsection{Esbozando una tipología}

Uno de los rasgos destacables de la investigación es la dificultad para establecer una tipología general del empresario pyme latinoamericano, pues no sólo se observa una gran heterogeneidad en sus negocios, en términos de activos, ventas, inversión, potencial (actividad) exportador(a), actividad económica, sino también en ellos mismos, pues hay una mezcla diversa en cuanto a nivel de educación, edad, experiencia (laboral y/o empresarial), género, entre otros aspectos.

Sin embargo, estas unidades económicas (las pymes), más diseminadas territorialmente respecto de las grandes empresas, muestran una tendencia a agruparse en los grandes centros urbanos: capitales y ciudades más importantes. Además, se encuentran dispersas, fundamentalmente, entre el comercio y los servicios, sin una preponderancia aparente (aunque en la visión por países se tiende a clarificar la orientación, sea al comercio o los servicios), con un peso claramente menor en la manufactura. La mayor parte de las empresas entrevistadas podrían conside- rarse como maduras, es decir, llevan más de 10 años en el mercado.

Con relación a los empresarios que las conducen, la edad promedio superaba los 40 años. Más del 60\% de los empresarios entrevistados tenía educación universitaria (completa o incompleta). En los países que contaban con información sobre años de experiencia, se observó que al menos la mitad de los entrevistados tenían más de 10 años en el campo empresarial. Finalmente, casi el 28\% de los empresarios entrevistados son mujeres. Esta característica contrasta con la de las pymes europeas, donde los empresarios son algo más jóvenes (35 años), y la proporción de mujeres es menor (20\%).

A pesar de esta «caracterización», la gran disparidad encontrada hace de este segmento una mezcla compleja de elementos que no puede ser sujeto de una sola política.

Por ello, y como una primera observación del estudio, los empresarios entrevistados señalan que las políticas públicas no han sabido establecer los énfasis adecuados ni llegar con eficiencia a la pyme, y en muchos casos han confundido (o agrupado) sus problemas con los de la microempresa, desarrollando acciones uniformes para estratos con problemas diferentes y que requieren, por tanto, de enfoques distintos.

\subsection{Las herramientas utilizadas}

La presente investigación toma como información de sustento las bases de datos, los análisis de contenido y las memorias de los focus groups y las entrevistas a profundidad, etcétera, de los diagnósticos realizados por Fundes en los países antes 
mencionados, además de fuentes de otras instituciones.

La investigación se desarrolla en dos fases: en la primera se analiza información de fuentes secundarias, estadísticas oficiales, investigaciones previas, mientras en la segunda se valida la información anterior mediante encuestas empresariales complementadas con focus groups y entrevistas en profundidad con actores relevantes.

A través de estas herramientas, se busca ahondar no sólo en el conocimiento de los actores involucrados en la problemática de las pequeñas y medianas empresas en los países en estudio, sino también identificar los puntos de vista de cada uno de ellos sobre las ocho áreas antes señaladas y, como resultado, establecer un orden de prioridad entre ellas.

Mediante el uso de la metodología de la doble triangulación ${ }^{2}$ entre fuentes de información (estudios previos y estadísticas, focus groups y entrevistas en profundidad) y entre actores (empresarios, gobierno, organizaciones intermedias), se pretende no sólo darle «voz» a los empresarios, quienes con frecuencia no han sido protagonistas de las políticas de fomento, sino también aumentar la consistencia de los resultados validando las percepciones de cada uno de los actores con las de los otros dos o estableciendo los puntos discordantes entre ellos.

En ese sentido, el estudio muestra - como caso general- un alto grado de acuerdo entre los actores sobre los temas

2. En este caso la triangulación se refiere tanto al uso de tres tipos de fuentes de información como a la consulta con tres tipos de actores. tratados, aunque no necesariamente sobre la importancia relativa de cada uno de ellos. La naturaleza de cada actor, su interacción en el mercado de servicios de desarrollo empresarial, su naturaleza política, entre otros aspectos, establecen un sesgo en su visión de los problemas y ayudan a entender las divergencias.

Desde la perspectiva establecida, hay concordancia general entre los ámbitos señalados en la primera fase y los resultados de la segunda fase. A pesar de ello, en los casos en que no hay coincidencia, la perspectiva temporal entre empresarios y otros actores involucrados parece ser la responsable. En general, se observa que son los analistas e investigadores quienes tienen una visión de largo plazo más arraigada con relación a los otros grupos analizados.

\section{La problemática regional}

Cada uno de los países que fue parte de la investigación generó -para cada fase del estudio-su propia lista de problemas. Ellos fueron señalados por los empresarios encuestados de una relación vasta y exhaustiva de problemas, desarrollada para cada país con base en las investigaciones previas disponibles. De las ocho áreas mencionadas líneas arriba, se identificaron tres, que por su relevancia y representatividad regional son pertinentes de ser presentadas: financiamiento, recursos humanos, y tecnología e información.

\subsection{El financiamiento}

En el ámbito del financiamiento encontramos varios elementos comunes pero también otros peculiares de cada realidad nacional. Siendo un tema de gran relevan- 


\section{Cuadro 5. Aspectos vinculados al financiamiento}

\section{Argentina}

- Exceso de garantías exigidas.

- Elevadas tasas de interés.

- Muchas deficiencias en el sistema financiero para evaluar a las pymes.

\section{Colombia}

- Altos costos crediticios.

- Ausencia de capital de riesgo.

- Ausencia de mecanismos como el factoring, el leasing, entre otros.

\section{Costa Rica}

- Ausencia de definición de sujetos de crédito.

- Requisitos y trámites excesivos.

- Altos gastos de formalización.

- Modalidades limitadas (factoring, leasing).

- Tasas de interés altas y plazos restringidos.

\section{Chile}

- Garantías excesivas para otorgar créditos.

- Elevadas tasas de interés.

- Altas comisiones.

- Mucho tiempo invertido en trámites.

\section{EI Salvador}

- Elevadas tasas de interés.

- Tipo de garantías exigidas.

- Exceso de requisitos.

- El sistema de evaluación de riesgo.

\section{Panamá}

- Garantías exigidas en respaldo del préstamo.

- Elevadas tasas de interés.

- Excesivos requisitos para ser sujetos de crédito.

- Altos costos de los trámites financieros.

- Plazos cortos para pagar el préstamo.

Elaboración propia con base en los diagnósticos desarrollados por Fundes.

cia en todos los países, llama la atención que -a diferencia de la percepción tradicional- no en todos sea el problema más importante. En otros casos, donde se pen- saría que no es problema, sí lo es, y con un amplio grado de acuerdo.

En el caso de Argentina, el acceso al financiamiento fue el obstáculo más comúnmente señalado por los empresarios entrevistados, y también el más importante. Los aspectos considerados más relevantes, en cuanto a este ámbito, son las garantías, las altas tasas de interés y la ausencia de mecanismos para evaluar los préstamos dirigidos a pymes (como puede verse en el cuadro 5). Otros factores de menor importancia fueron la falta de líneas específicas para pymes, el exceso de exigencias formales por parte de los bancos y los elevados gastos administrativos para la tramitación de créditos.

En Colombia, el acceso al financiamiento es el tercer obstáculo más importante para los empresarios (luego de la violencia y los impuestos). En esta área, al igual que en Argentina, los siguientes se revelan como los aspectos más sensibles de la problemática: las garantías (insuficientes), los altos costos crediticios, los altos costos del sistema financiero (asociados a la relación entre el tamaño del crédito pyme y los costos fijos de los bancos, la concentración de los préstamos en los conglomerados empresariales, etcétera), la ausencia de capital de riesgo (por la carga tributaria para los inversionistas, el marco legal restrictivo, entre otras razones), la ausencia de mecanismos como el factoring y el leasing (los cuales tienden a no resultar debido al riesgo asociado, los pequeños montos y la poca información disponible), entre otros factores.

En Costa Rica, el tema del acceso al financiamiento aparece en el tercer lugar de las prioridades de los empresarios, después de las elevadas cargas sociales y la 
ilegalidad. Por el lado de la oferta, se observa la existencia de fuentes de financiamiento disponibles para las pymes; sin embargo, se reconoce la presencia de altos costos fijos en el sistema financiero que encarecen el crédito y, por tanto, su colocación. Además, no hay una definición homogénea para identificar a los sujetos de crédito, los trámites de aprobación son lentos (45 días sólo para obtener la documentación requerida por el sistema) y los gastos de formalización son altos (legalización de documentos, peritajes, etcétera). Como en los países anteriores, el nivel de garantías reales es alto y no existe el concepto de cliente (no hay memoria sobre este), ya que cada nueva operación de un mismo cliente pasa por el mismo trámite regular.

En el caso de Chile, el financiamiento como obstáculo es considerado en el cuarto lugar de la jerarquía, luego de la competencia desleal, el funcionamiento del aparato estatal y la situación macroeconómica general. El problema no es tanto el acceso al financiamiento, sino las condiciones en que es otorgado. Lo más crítico para los empresarios son las garantías y las tasas de interés. Con menor frecuencia fueron mencionados las altas comisiones, el tiempo invertido en los trámites y la falta de información. Estos obstáculos fueron señalados como causales de pérdida de negocio, retraso o cancelación de proyectos. Otro hecho relevante señalado por los empresarios entrevistados fue que su principal fuente de financiamiento es el capital propio.

En El Salvador, el tema del acceso al financiamiento recibió el segundo lugar en la jerarquía de los problemas, sólo después de las condiciones de la competencia interna (básicamente, poder de mer- cado y competencia desleal). Los aspectos más sensibles de la problemática fueron las elevadas tasas de interés, el tipo de garantías exigidas (hipotecaria de la empresa, hipotecaria de una propiedad personal, firma solidaria, bienes muebles, fiadores, etcétera) y los requisitos excesivos. Otros obstáculos de menor jerarquía fueron el sistema de evaluación del riesgo crediticio, los plazos otorgados para la cancelación de los créditos, entre otros.

En Panamá, las condiciones de acceso al financiamiento ocuparon el primer lugar en la problemática de las pymes. Este resultado encierra una aparente paradoja, pues la percepción inicial de $\mathrm{Pa}$ namá como uno de los centros financieros más importantes de América haría suponer que el tema del financiamiento no es un problema en ese país. El mensaje, al parecer, es que no se cuenta con un diseño adecuado de financiamiento para las pymes.

En este caso, los problemas señalados por los empresarios como los más importantes fueron las elevadas garantías (entre el $100 \%$ y el $150 \%$ del monto del crédito solicitado), las tasas de interés (entre $11 \%$ y $15 \%$ anual $^{3}$ ) y los requisitos excesivos (estados financieros auditados de por lo menos los dos últimos años de operaciones, avalúo de propiedades, estudios de factibilidad, entre otros).

Como puede apreciarse, el tema del acceso al financiamiento tiene lugares comunes en las tasas de interés, las garantías y los requisitos. Otros elementos

3. Cabe señalar que la moneda de curso legal en Panamá es el dólar estadounidense, por lo que todas las transacciones y, por supuesto, las tasas de interés se refieren a esta moneda. 
menos señalados, pero importantes, son los sistemas de evaluación del riesgo y los costos de los trámites financieros.

Lo anterior muestra indirectamente varios aspectos de la problemática. Por un lado, en muchos casos las pymes no cumplen los requisitos comúnmente solicitados por los bancos. Este problema de información supone la necesidad de contar con otros mecanismos de difusión, de modo que tanto la oferta de financiamiento como las condiciones del mismo lleguen, efectivamente, a los potenciales clientes.

Al mismo tiempo, el poco grado de formalidad de la gestión administrativa en varias de estas empresas es un factor limitante para el acceso al financiamiento. En este punto, la responsabilidad de los empresarios es clara.

Adicionalmente, nos encontramos con un sistema financiero donde no existen o son inoperantes los sistemas de evaluación de empresas de menor tamaño, o para montos pequeños de préstamo. Este es un indicador del poco interés del sistema financiero por este mercado, pero también de su propia ineficiencia para captar otros segmentos donde el volumen de clientes es más importante que el monto individual de los créditos. Esto supone el desarrollo de sistemas más adecuados para clientes pequeños, pero también un incremento de la eficiencia del sistema financiero.

\subsection{Recursos humanos}

En el tema de los recursos humanos se combinan las propias necesidades de los empresarios por personal calificado y experimentado, la oferta existente de capa- citación y consultoría y las restricciones -que los empresarios manifiestan- que las regulaciones laborales (y otras asociadas) establecen en las relaciones de mercado.

En este sentido, Argentina es un caso particular. Si bien el tema de los recursos humanos es abordado desde la perspectiva de la regulación laboral, la consultoría y la capacitación, y las calificaciones y competencias del recurso humano en la empresa, estos problemas no aparecen sino en el puesto diez de la jerarquía establecida por los empresarios, bajo la forma de «servicios de capacitación y consultoría inadecuados a las necesidades empresariales». Sin embargo, no por ello el tema debe ser considerado algo menor, ya que en el análisis más específico los recursos humanos fueron señalados como de significativa importancia, sobre todo en lo referido a legislación laboral, consultoría y capacitación, y demanda de recursos humanos por las pymes.

En términos de legislación laboral, la «precarización» o deterioro del empleo ha sido, en la práctica, resultado de acciones como la fragmentación de las vacaciones y/o del aguinaldo, la incorporación de personal con costos nulos de despido, la flexibilización de la jornada laboral y/o de las funciones de los trabajadores; medidas que logran entre los empresarios entrevistados un grado de aprobación superior al $60 \%$.

En cuanto a los aspectos de consultoría y capacitación, se dividió a los empresarios en dos grupos: quienes recibieron consultoría o capacitación y quienes no lo hicieron. Quienes no recibieron acciones de este tipo revelan un menor dinamismo y sus empresas se observan como las de menor tamaño. Los que sí recibieron 
capacitación o consultoría no sólo tienen empresas más grandes, sino también más dinámicas. Al ser consultados quienes no habían adquirido consultoría o capacitación señalaron que son muy caras, el nivel técnico no es adecuado, están hechas para empresas de mayor tamaño, no las necesitan, entre otras razones. Por el contrario, quienes sí adquirieron estos servicios opinaron que la calidad en general es buena.

Para propósitos del análisis, la demanda de recursos humanos por parte de las pymes fue dividida en dos categorías: superiores (gerentes y supervisores) y subalternos (administrativos y operativos). En ambos casos lo que buscan las pymes es, básicamente, experiencia previa y potencialidad para identificar y solucionar problemas. Se reconoce que ha aumentado la exigencia respecto a competencias laborales; sin embargo, las mismas empresas que han incrementado sus estándares son las que enfrentan mayores dificultades para conseguir personal calificado.

En Colombia, el tema de los recursos humanos, específicamente la falta de personal calificado, ocupó el sétimo lugar en la jerarquía, después de la violencia, los impuestos, el acceso al crédito, el contrabando, las prestaciones sociales y los servicios públicos. Sin embargo, el tema de las prestaciones sociales como parte del ámbito de los recursos humanos ocupó la quinta posición. En términos de lo que representan las cargas sociales en Colombia, el estudio señala que las empresas enfrentan cargas equivalentes, aproximadamente, al $52 \%$ del salario básico del trabajador. Este sería el mayor obstáculo para los empresarios dentro del contexto laboral.

Conviene señalar que la carencia de personal calificado rebasa el ámbito de las pymes y coloca en el debate aspectos como el rezago educativo, la deserción escolar y la inversión del Estado en la educación básica. En términos de la educación técnica, el Servicio Nacional de Aprendizaje (Sena), como principal institución de capacitación técnica y asesoría tecnológica, no cumple con su función en la medida en que se encuentra orientada, principalmente, a prestar asistencia a las grandes empresas. No tiene mucha interacción con las asociaciones empresariales, su administración es pública aunque su financiamiento proviene de la empresa privada, y sus costos son excesivos: la hora de capacitación promedio cuesta 42 dólares, mientras su símil en Ecuador maneja costos de 11 dólares por hora. De esta forma, la oferta de capacitación no se encuentra a la altura de los desafíos actuales.

Costa Rica, al igual que Argentina, muestra en el tema de los recursos humanos varias dimensiones. Por un lado, las cargas sociales encabezan la jerarquía de los obstáculos al desempeño competitivo de las pymes; y por otro lado, el aspecto de la consultoría y la capacitación ocupa el último lugar en la escala de 15 obstáculos.

Las cargas sociales han sido ampliamente documentadas como uno de los aspectos más gravosos para los empresarios. De hecho, las contribuciones patronales a la Caja Costarricense del Seguro Social (CCSS) son consideradas un indicador del grado de formalidad o informalidad de las empresas, debido a su alto costo.

En cuanto a la capacitación y consultoría, investigaciones de Fundes Costa Rica (2000) señalan que aproximadamente el $87 \%$ de las pymes ha participado en 
cursos de formación. Sin embargo, la queja generalizada es que estos cursos no responden a las necesidades de las pymes, tratan aspectos muy generales de la problemática empresarial. De la misma forma, sobre la consultoría -contratada por el $57 \%$ de las pymes- se opina que es una herramienta costosa, demasiado específica y cuyos resultados no han sido los deseados.

El tema de los recursos humanos en Chile se aborda también desde tres diferentes perspectivas: la legislación laboral, la calificación de los recursos humanos y la calidad de la oferta de capacitación. La legislación laboral ocupó la quinta posición, luego de la competencia desleal, el aparato estatal, la situación macroeconómica y las condiciones de financiamiento. La calidad de los recursos humanos ocupó la sétima posición y, finalmente, la calidad de la oferta de capacitación se ubicó en la posición número catorce (de dieciséis obstáculos).

En opinión de los empresarios entrevistados, la legislación laboral les genera costos significativos, especialmente los asociados al despido de trabajadores. Otras consideraciones señaladas son los costos de contratar y las cargas sociales. Respecto de la disponibilidad, calidad y costos de los recursos humanos, aunque es una variable considerada como de moderada influencia en el desempeño de las pymes, se observa que la falta de compromiso y la insuficiente capacitación de los trabajadores son elementos relevantes de la problemática de los recursos humanos.

Finalmente, en cuanto a la capacitación, la falta de tiempo y los costos son los obstáculos señalados con mayor énfasis por los empresarios. De la misma for- ma, hay un problema de información respecto de la oferta disponible, la calidad de la capacitación y la burocratización del Servicio Nacional de Capacitación y Empleo (Sence); aspectos que deben tomarse en cuenta.

En el caso de El Salvador, aparecen los mismos tres temas pero con una menor importancia. De hecho, la calificación de los recursos humanos es el octavo en la jerarquía, los servicios de capacitación y consultoría ocupan el décimo lugar y la legislación laboral figura en el último puesto de la problemática. Al parecer, en la percepción de los empresarios entrevistados, los recursos humanos no son un problema apremiante. Sin embargo, tampoco puede decirse que sean algo trivial.

Sobre el tema de la calificación, se separan las necesidades de operarios, personal técnico y gerencial. En cuanto a los operarios, la experiencia previa es el aspecto central; le sigue la capacidad de trabajar en equipos y la formación técnicoprofesional. En el caso del personal técnico, la formación y la experiencia previa son aspectos clave. Finalmente, respecto de los cuadros gerenciales, la capacidad para tomar decisiones, la experiencia previa, la creatividad y la innovación son los aspectos medulares de la demanda.

Respecto de los servicios de capacitación y consultoría, existe un conocimiento relativo de la oferta. Sin embargo, no más de dos de cada cinco empresarios han utilizado algún servicio de este tipo. El aspecto sobresaliente de estos servicios es que quienes han recibido algunos de ellos los valoran positivamente. Finalmente, en cuanto a la legislación, las cotizaciones a las AFP (administradoras de fondos de pensiones), al Instituto Salvadoreño del 
Seguro Social (ISSS) y los pagos por indemnización son los aspectos mayormente señalados por los empresarios.

Finalmente, en Panamá el tema de los recursos humanos se percibe desde la perspectiva de la legislación laboral, la disponibilidad del recurso humano y la capacitación y consultoría. En efecto, los costos de despido, materia de la legislación laboral, ocuparon el cuarto puesto en la jerarquía de problemas, mientras que la obtención de mano de obra calificada ocupó la sexta posición; las obligaciones asociadas a la contratación de personal, la octava posición; el costo de las prestaciones laborales, la décima; y finalmente, la disponibilidad de servicios de capacitación adecuados, el puesto duodécimo (de diecisiete).

En cuanto a la legislación laboral, los aspectos mayormente mencionados son los trámites para el despido, las indemnizaciones por despido de personal, las horas extraordinarias, los salarios y las contrataciones. Se considera que la legislación laboral es rígida, contribuye a incrementar los costos y, en consecuencia, desalienta la generación de empleo. De esta forma, la legislación laboral no parece ajustarse a la realidad de las pymes.

La calidad de los recursos humanos fue evaluada según las características deseadas para los tres niveles ocupacionales: técnico, ejecutivo y operario. En el primer caso, la experiencia previa y la formación fueron los dos principales requerimientos. En cuanto a los ejecutivos, también la experiencia previa y la capacidad de identificar, diagnosticar y solucionar problemas fueron los más relevantes. Finalmente, en el caso de los operarios, la experiencia previa y el saber leer, redac- tar, expresarse y escuchar fueron las demandas más frecuentes de los empresarios. Asimismo, se considera que la mano de obra local es cara, poco calificada y poco productiva con respecto a otros países de la región.

Comparando los resultados de los países en estudio, hay tres aspectos relevantes que deben tomarse en cuenta. El primero es la legislación laboral, que en todos los casos se considera rígida y atentatoria contra la generación de empleo. Sin embargo, se observa también la emergencia de mecanismos «alternativos» de contratación, lo cual supone, en la práctica, la flexibilización y, al mismo tiempo, la precarización del empleo. Valdría la pena reflexionar si esta es la mejor solución para los trabajadores, frente a una posible modificación de la legislación laboral vigente.

El segundo aspecto relevante guarda relación con la calidad del recurso humano. En muchos casos se hace referencia a la poca calificación (teórica y práctica) del personal. Esto tiene que ver, por un lado, con la formación básica y, eventualmente, técnica y, por otro lado, con la experiencia previa. Claramente se observa que existe un divorcio entre la formación y las necesidades del mercado laboral, razón por la cual es clave en este aspecto el papel del sistema educativo y de las propias empresas. La experiencia laboral es el otro factor preponderante. Sin embargo, no puede haber experiencia si no hay trabajo previo. Para ello es necesario generar (o generalizar) mecanismos del tipo escuela-empresa, que permitan a los profesionales técnicos adquirir la experiencia necesaria en sus últimos años formativos a fin de que puedan ingresar con éxito al mercado laboral. 


\section{Cuadro 6. Aspectos vinculados a los recursos humanos}

\section{Argentina}

- Convenios laborales muy rígidos.

- Escasas competencias laborales del recurso humano.

- Mecanismos de consultoría y capacitación poco apropiados.

\section{Colombia}

- Cargas sociales.

- Otras disposiciones laborales.

- Falta de personal calificado.

\section{Costa Rica}

- Elevadas cargas laborales.

- Ausencia de oferta relevante de consultoría y capacitación.

\section{Chile}

- Legislación laboral

- Costos de despedir.

- Costos de contratar.

- Calidad del recurso humano

- Falta de compromiso.

- Falta de calificación.

- Calidad de la oferta de capacitación

- Falta de tiempo y altos costos de capacitación.

- Falta de información.

- Burocracia del Sence.

\section{El Salvador}

- Calidad de recurso humano

- Experiencia previa.

- Capacidad técnica.

- Capacidad para tomar decisiones.

- Capacitación y consultoría

- Falta de idoneidad para cumplir los perfiles ocupacionales.

- Bajos niveles de inversión en capacitación.

- Altos costos laborales derivados de la legislación vigente.

- Legislación laboral

- Cotizaciones a las aseguradoras de fondos de pensiones (AFP).

- Cotizaciones al Instituto Salvadoreño del Seguro Social (ISSS).

- Pagos por indemnizaciones.

\section{Panamá}

- Legislación laboral

- Trámites de despido.

- Indemnizaciones por despido de personal.

- Horas extraordinarias.

- Recursos humanos

- Experiencia previa.

- Formación técnica y/o profesional.

- Capacidad de interacción.

Elaboración propia con base en los diagnósticos desarrollados por Fundes. 
Finalmente se encuentra el tema de la capacitación, vinculado con el de la calidad del recurso humano. En los países donde se tomó en cuenta este factor, los aspectos críticos fueron los costos, el tiempo requerido y la información sobre el servicio. Particularmente relevante fue que los empresarios entrevistados que vivieron la experiencia de la capacitación tienen, en general, una opinión positiva de esta. Hay un problema de información implícito que deben resolver los prestadores de servicios, el Estado y otras organizaciones intermedias: el sistema financiero, las cámaras, los gremios, entre otros.

No obstante, los elevados porcentajes de aprobación de los servicios de capacitación y/o consultoría son una señal positiva, ya que el problema de información que impide el acceso a los servicios puede compensarse con un buen servicio ofrecido, el cual repercutirá en la comunidad empresarial por medio del «boca a boca». Cabe analizar si el impacto de estos servicios sobre el empleo, las ventas y el producto es también significativo; en todo caso, la capacitación es una primera variable que debe complementarse con la evaluación de su impacto. También sería importante considerar la necesidad de un «subsidio» a la primera experiencia en consultoría y/o formación, a manera de un «simulacro». La primera experiencia exitosa sería un mecanismo de generación de información sobre estos servicios, y de construcción de un mercado de servicios de desarrollo empresarial.

Como corolario de este tema, y en términos de la propia realidad empresarial, el hecho que algunas empresas no consideren como relevantes para ellas la consultoría ni la capacitación puede deberse a que: a) no les interesa, por experiencias negativas en el pasado del empresario o de su entorno; b) realmente no tienen un referente de lo importante que pueden ser para su desarrollo; y c) son empresarios que tienen un techo de desarrollo definido del cual no piensan salir.

\subsection{Tecnología e información}

Este es un tema particularmente relevante para el desarrollo competitivo de las empresas. Sin embargo, el fomento tecnológico a todo nivel, aunque indispensable, no siempre es tomado en cuenta apropiadamente, debido a que otros factores de la problemática empresarial, más urgentes y factibles de resolver, tienden a tomar su lugar.

En el caso de Argentina, el tema tecnológico no aparece explícitamente en la jerarquización de la problemática vista desde la perspectiva empresarial. Los resultados de la encuesta parecen señalar cierto desinterés por los aspectos asociados a la creación de capacidades y de ventajas competitivas de mediano plazo. Ello sería un signo de las características preponderantes de este grupo empresarial en ese país. Sin embargo, el tema de la información es horizontal a todas las áreas, en la medida en que su déficit es importante -sea porque no existe o porque no se canaliza adecuadamente- e impide la toma de decisiones en muchas áreas, incluida la tecnológica.

Colombia, al igual que Argentina, no manifiesta explícitamente el tema tecnológico en la jerarquía de los problemas. Sin embargo, el acceso a la tecnología aparece como un área problemática dentro de la estructura general de problemas a través de varios canales. Uno de ellos es el sistema educativo. En la medida en 
que este no responde a las necesidades empresariales, la aportación práctica del capital humano a la mejora tecnológica se reduce. De igual forma, el acceso al crédito dificulta a las pymes la adquisición de tecnología. Finalmente, la carencia de información y asesoría respecto de las innovaciones tecnológicas y su aplicabilidad a las empresas es una restricción adicional a la mejora tecnológica.

En el caso de Costa Rica, el tema tecnológico aparece, por un lado, como parte del sistema de apoyo a las pymes y, por otro lado, en la infraestructura. En la jerarquía de obstáculos, estos aspectos son mencionados en el cuarto y quinto lugar. $\mathrm{Al}$ respecto, la necesidad de contar con un sistema de apoyo a las pymes que involucre tecnología e información es algo perentorio para los empresarios costarricenses. La ausencia de mejoras en la tecnología se asocia también a su alto costo y a la falta de instancias asesoras (información) e instituciones que acerquen o faciliten la oferta tecnológica. En este mismo sentido, la cooperación entre empresas y centros de investigación puede ser parte de una solución integradora para eliminar el rezago tecnológico.

La situación de Chile frente el tema tecnológico es interesante. Aunque se señala el acceso a la tecnología como el undécimo tema en la agenda de problemas (de un total de dieciséis), vale la pena resaltar que este asunto es más importante para la mediana que para la pequeña empresa, pues la primera ubica el tema en el octavo lugar de la jerarquía.

En este país, a diferencia de otros casos, se observa una visión positiva de los entrevistados acerca de la capacidad de innovación de las empresas. Sin embargo, desde el punto de vista de los obstáculos, el costo de la tecnología es el factor limitante más importante para su adopción. Otros aspectos menos señalados -pero importantes- son la falta de información y de servicios complementarios, la ausencia de capacidad de negociación e, incluso, el hecho que la tecnología disponible en el mercado no es útil a la empresa.

Un factor importante que debe señalarse es la presencia de una gran heterogeneidad en las posibilidades de innovación entre las empresas de dentro y fuera de la región metropolitana, que favorece claramente a las empresas metropolitanas. Asimismo, la mayor capacidad de innovación se asocia a las empresas exportadoras frente a las que no exportan.

En El Salvador, el tema tecnológico ocupa el sétimo lugar en la jerarquía de un total de once puntos considerados como los más problemáticos para las pymes. De los aspectos considerados clave, la conclusión rotunda de los empresarios es que la tecnología disponible no responde a las necesidades de las pymes. De hecho, menos de la mitad de los entrevistados considera que la tecnología empleada en las áreas administrativa, productiva y de ventas es avanzada; la mayoría manifiesta que es tradicional.

De esta manera, puede decirse que la tecnología empleada por las pymes es obsoleta. Los bajos niveles de investigación y desarrollo existentes y la falta de una cultura de calidad son factores que no favorecen la adopción o difusión tecnológica. Igualmente, los altos costos de adquisición de nueva tecnología, las dificultades para obtener financiamiento y la insuficiente calificación de los recursos humanos para dar el salto tecnológico 
inciden en la escasa adopción de nuevas tecnologías. Finalmente, la ausencia de información sobre lo disponible y factible para los requerimientos de las pymes también se vuelve un obstáculo para el desarrollo competitivo de este sector empresarial.

Para terminar, en Panamá el tema tecnológico se encuentra en el último lugar (17) entre las prioridades de las pymes. Sin embargo, los hallazgos de la investigación indican que: a) un porcentaje sig-

\section{Cuadro 7. Aspectos vinculados a la tecnología e información}

\section{Argentina}

- Falta de intermediarios para vincular las necesidades con las instituciones de innovación.

- Falta de desgravaciones impositivas que alienten la innovación tecnológica.

\section{Colombia}

- Problemas de acceso a tecnología (económicos, de información, etcétera).

\section{Costa Rica}

- Inadecuada infraestructura tecnológica.

\section{Chile}

- Alto costo de la tecnología

- Falta de información.

- Escasa utilidad de la tecnología disponible.

- Falta de capacidad de negociación.

- Falta de servicios complementarios.

\section{EI Salvador}

- Alto costo de la tecnología.

- Financiamiento poco disponible.

- Escasa información sobre tecnología.

- Ausencia de asesoría.

\section{Panamá}

- Alto costo de la tecnología.

- Difícil acceso al financiamiento.

- Impuestos de introducción.

- Escaso personal calificado. nificativo de estas empresas emplea tecnología tradicional; b) quienes invierten en tecnología son una proporción mínima; c) los altos costos de la tecnología son una de las razones de esta deficitaria inversión; d) no hay clara conciencia de los beneficios de la adopción de nueva tecnología; y e) este fenómeno es más recurrente en las pequeñas empresas que en las medianas.

Por los resultados observados, puede advertirse cómo el tema tecnológico es uno de los que ha causado mayor inquietud entre los investigadores. La hipótesis implícita en cada país fue que este era un aspecto considerado clave por los empresarios para su desempeño competitivo en el mediano y largo plazo. Sin embargo, el resultado general respecto de la poca importancia relativa que se le otorga al tema de la tecnología e información frente a otros como el financiero no es casual ni muestra irracionalidad en las respuestas empresariales. La visión argentina de cierta forma nos ayuda a entender las razones de este hecho. Podríamos decir que el empresario pyme tiene un horizonte de planeación muy corto, aspecto que, sin embargo, no tiene que ver con una actitud rentista, aunque sí cortoplacista. Más bien, el entorno de negocios en el que se desenvuelve juega permanentemente en su contra. En este punto es importante retomar el tema de las instituciones y de la variabilidad de las políticas económicas y de las políticas en general.

\subsection{El rol de la interrelación y la estabilidad}

Muchos de los problemas asociados a las áreas señaladas en este estudio se entrecruzan entre sí, a veces de manera directa y otras indirectamente. Otros se encuentran asociados a la ausencia de estabili- 
dad en las políticas de algunos de los países considerados. En todo caso, la interrelación y la ausencia de estabilidad han sido elementos comunes en la problemática estudiada.

Tal como señalan Gonzales de Olarte y Samamé (1991) para el caso peruano -aunque puede generalizarse para los otros países latinoamericanos-, durante los últimos treinta años ha habido cambios pendulares en la orientación de las políticas económicas de un gobierno a otro, e incluso dentro de un mismo gobierno. Se pasó de la «ortodoxia» a la «heterodoxia»y viceversa, lo que contribuyó a generar inestabilidad estructural en el sistema económico.

A priori, parece plausible afirmar que estos vaivenes en las políticas hayan influido sobre el comportamiento de los grandes agregados. Es decir, las oscilaciones macroeconómicas experimentadas en América Latina se relacionan con las variaciones en la política. La hipótesis tiene que ver con la inestabilidad política y económica en los países en desarrollo, particularmente los de América Latina. A su vez, la interrelación de muchos de estos ámbitos genera que esa inestabilidad se «contagie» y cause la presencia de políticas «pendulares», que responden a los gobiernos de turno y a las estructuras de incentivos que ellos promueven. Ello origina un incremento en la inestabilidad e incertidumbre en los agentes económicos. La consecuencia de todo ello es la mayor dificultad en la planeación de la inversión de largo plazo. Esto se refleja más que en la media, en la varianza del PBI real, tal como puede apreciarse en el cuadro 8 .

Esta variabilidad (pendularidad) en las políticas conduce al incremento de la inestabilidad y la incertidumbre, factores relevantes en la decisión de inversión de empresas e individuos. Los entornos económico-políticos inestables imponen «restricciones institucionales» variables, $\mathrm{y}$ posiblemente aleatorias a través del tiempo, lo que incrementa los costos de información y de planeación pertinentes para las decisiones de inversión. Esta es una

\section{Cuadro 8. Media y desviación estándar de la tasa de crecimiento} del PBI real para países seleccionados (1961-1996)

\begin{tabular}{lcc}
\hline Países & Media & Desviación estándar \\
\hline Estados Unidos & 3,02 & 2,13 \\
Alemania & 2,95 & 2,27 \\
Reino Unido & 2,31 & 2,13 \\
Promedio de los desarrollados & $\mathbf{2 , 7 6}$ & $\mathbf{2 , 1 8}$ \\
Argentina & 2,35 & 5,37 \\
Chile & 4,09 & 5,62 \\
Costa Rica & 4,59 & 3,60 \\
Promedio de los en desarrollo & $\mathbf{3 , 6 8}$ & $\mathbf{4 , 8 6}$
\end{tabular}

Fuente: Emilio Cevallos. Tendencia y variabilidad en el desempeño económico; análisis empírico comparativo de países desarrollados versus subdesarrollados. México, ITAM. 1998. Tesis de maestría en Economía. 
respuesta factible a la imposibilidad de planeación que deriva en una visión cortoplacista por parte de los empresarios de las pymes. Por ello, los problemas de corto plazo parecerían ser los únicos que el empresario considera lo suficientemente importantes como para invertir dinero y tiempo en ellos, especialmente en un contexto de escasez de ambos recursos.

\section{Algunas reflexiones sobre los resultados: obstáculos al desarrollo de las pymes en América Latina}

Más allá de los temas específicos abordados en este avance, es conveniente cerrar el artículo con algunas reflexiones generales sobre la investigación. Una de las primeras es acerca del carácter heterogéneo de los actores pyme. Factores como el tamaño de la empresa, la edad del empresario, su experiencia (laboral y empresarial), el carácter familiar (o no) de la unidad económica, el sector en el que se desarrolla, su ubicación espacial, la relación que mantiene con otros actores (empresarios, gobierno, sistema financiero, clientes, etc.), su dotación de capital, la infraestructura que posee, su grado de avance tecnológico, entre otros, permiten calificar a este segmento empresarial como un grupo marcado por las diferencias. A ello se suman las acciones de los gobiernos que para definirlos usan los más variados criterios y denominaciones.

La incomprensión por parte de los gobiernos de esta heterogeneidad ha llevado a considerar a la pyme, e incluso a la mipyme (microempresa, pequeña y mediana empresa) como un conjunto homogéneo de empresas. Puesto que las políticas de fomento se han guiado por este signo, la consecuencia ha sido su ausencia de impacto, cuando no la falta de credibilidad de las acciones públicas y, por tanto, su limitado alcance en términos de beneficiarios. Al mismo tiempo, esto ha servido para que algunas empresas, más dinámicas e informadas que el promedio, se aprovechen de la situación monopolizando los recursos y la ayuda.

La segunda reflexión de importancia guarda relación con el carácter sistémico de la problemática de las pymes. En efecto, cada uno de los ámbitos en los que los empresarios señalan oportunidades de mejora se configura, a su vez, en problemáticas que se interrelacionan entre sí, horizontalmente, e incluso involucran a otras áreas que van más allá de las especificidades de una investigación sobre el entorno de las pymes.

Podemos señalar -por ejemplo- la multidimensionalidad de la relación entre la capacitación laboral, la educación, la tecnología y el financiamiento. Por supuesto, la ausencia de financiamiento limita cualquier intento de mejora, sea en el desarrollo de competencias laborales o en la adopción de tecnología -que requiere del desarrollo de nuevas competencias laborales-. Asimismo, las limitadas capacidades de los trabajadores no tienen que ver sólo con su formación laboral, sino con el tipo de educación recibida. Esta limitación reduce las posibilidades de absorber nuevos conocimientos (asociados a la tecnología moderna), por lo que las restricciones educativas se convierten en limitaciones para la adopción tecnológica.

La relación entre el Estado y las empresas es otro asunto de relevancia crítica. Tanto en los temas de tecnología, recursos humanos, financiamiento y otros, las condiciones en que la interacción se 
produce es crucial para el entendimiento tanto de la conducta pública como de la respuesta empresarial.

De lo anterior se desprende la trascendencia del entorno en el desempeño competitivo de las empresas. Por ello, el desafío central de la región es implementar políticas resultado de acciones coordinadas, establecer definiciones concertadas y definir los roles que cada uno de los actores económicos debe jugar en el desarro1lo. La pyme, como cualquier otro actor económico, recibe señales y actúa en consecuencia.

En este sentido, la tercera reflexión advierte acerca del impacto de las políticas sobre el desempeño económico y de cómo la variabilidad de estas a lo largo del tiempo ha desarrollado una configuración empresarial desconfiada y sin perspectivas de largo plazo.

Este es un llamado de atención en pos de la promoción de la estabilidad y la consistencia de las políticas económicas, y de las políticas en general. En los escenarios donde los vaivenes de la política no inciden en la conducta económica, como en los países europeos, se mantiene una notable estabilidad económica a pesar de los conflictos existentes en el orden político. Por ello, el mayor desarrollo institucional debe ser la antesala del apartamiento entre las decisiones políticas y las medidas económicas, y el referente de conductas empresariales más «emprendedoras». 


\section{Bibliografía}

BRAVO, David; CRESPI, Gustavo y GUTIÉRREZ, Irma. 2002.El desarrollo se escribe con pyme: el caso chileno, desafíos para el crecimiento. Santiago: Cepal, Universidad de Chile y Fundes Chile.

CASTILLO, Geovanny y CHAVES, Luis. 2001. Pymes: una oportunidad de desarrollo para Costa Rica. San José: Fundes Costa Rica.

2000. Obstáculos al desarrollo de las pymes. San José: Fundes Costa Rica.

CEPAL. 2003. Balance preliminar de las economías de América Latina y el Caribe 2003. En [www.cepal.cl].

FUNDES Costa Rica. 2000. Necesidades de formación y consultoría de las pequeñas y medianas empresas en Costa Rica: un análisis de los servicios que ofrece Fundes Costa Rica. San José: Fundes Costa Rica.

GONZALES DE OLARTE, E. y SAMAMÉ, L. 1991. El péndulo peruano, políticas económicas, gobernabilidad y desarrollo 1963-1990. Lima: Instituto de Estudios Peruanos.

HOWALD, Félix. 2001. Obstáculos al desarrollo de la pyme causados por el Estado: el caso del sistema tributario en Colombia. Santiago: McGraw Hill Interamericana y Fundes.
MARTÍNEZ, Julia y VIÉYTEZ, Elcira. 2002. Desafios y oportunidades de las pymes salvadoreñas; construyendo una agenda de desarrollo. San Salvador: ANEP, Fundes y Fundapyme.

PINILLA, Silma. 2002. Una oportunidad para Panamá: remover los obstáculos al desarrollo de las pymes. Guatemala: Fundes Panamá.

RODRÍGUEZ, Astrid. 2003. La realidad de la pyme colombiana; desafío para el desarrollo. Bogotá: Fotolito Colombia Preprensa Digital.

UNITED NATIONS DEVELOPMENT PROGRAM. 2004. Human development report. En [www.undp.org].

YOGUEL, Gabriel y MOORI-KOENIG, Virginia (coords.). 1999. Los problemas del entorno de negocios; el desarrollo competitivo de las pymes argentinas. Buenos Aires: Fundes Argentina y Miño y Dávila Editores.

ZEVALLOS, Emilio. 2003. Micro, pequeñas y medianas empresas en América Latina. Revista de la Cepal. Santiago, abr., n. ${ }^{\circ} 79$.

ZEVALLOS, Emilio y VELAZCO, Enrique. 2003. Construyendo el desarrollo a través de las pymes: la experiencia boliviana. San José y La Paz: Buendiseño Comunicación Visual. 